\title{
Prognostic evaluation of resectable colorectal cancer using platelet-associated indicators
}

\author{
WEIHUA QIAN $^{1,2^{*}}$, XIN-XIN GE ${ }^{1 *}$, JING WU $^{1 *}$, FEI-RAN GONG ${ }^{3}$, MENG-YAO WU $^{1}$, \\ MENG-DAN XU ${ }^{1}$, LIAN LIAN ${ }^{1,4,5}$, WEN-JIE WANG ${ }^{1,6}$, WEI LI $^{1,4,7}$ and MIN TAO ${ }^{1,7}$ \\ ${ }^{1}$ Department of Oncology, The First Affiliated Hospital of Soochow University; ${ }^{2}$ Department of Oncology, \\ Zhangjiagang Hospital of Traditional Chinese Medicine Affiliated to Nanjing University of Chinese Medicine; \\ ${ }^{3}$ Department of Hematology, The First Affiliated Hospital of Soochow University, Suzhou, Jiangsu 215006; \\ ${ }^{4}$ Comprehensive Cancer Center, Suzhou Xiangcheng People's Hospital; ${ }^{5}$ Department of Oncology, \\ Suzhou Xiangcheng People's Hospital, Suzhou, Jiangsu 215000; ${ }^{6}$ Department of Radio-Oncology, \\ The Affiliated Suzhou Hospital of Nanjing Medical University, Suzhou, Jiangsu 215001; ${ }^{7}$ PREMED Key \\ Laboratory for Precision Medicine, Soochow University, Suzhou, Jiangsu 215021, P.R. China
}

Received March 8, 2018; Accepted April 8, 2019

DOI: $10.3892 / \mathrm{ol} .2019 .10388$

\begin{abstract}
Colorectal cancer (CRC) represents the third most common malignancy worldwide. The aim of the present study was to investigate the predictive values of platelet-associated indicators, including platelet count (PLT), plateletcrit (PCT), mean platelet volume (MPV) and platelet distribution width (PDW) in patients with resectable CRC. The current retrospective study included 153 patients who were pathologically diagnosed with resectable CRC. The patients were divided into two groups according to the median value of PLT, PCT, MPV or PDW. To evaluate the changes in PLT, PCT, MPV and PDW following resection and adjuvant chemotherapy, the concept of post-/pre-treatment PLT, PCT, MPV and PDW ratios was introduced, where $<1$ indicated decreased PLT, PCT, MPV and PDW values after treatment, and where $\geq 1$ suggested stable or increased values. It was revealed that a low MPV prior to treatment correlated with a higher tumor stage. Surgery significantly decreased MPV, but had no impact on PLT, PCT or PDW. Adjuvant chemotherapy significantly decreased PLT and PCT, increased MPV and had no effect on PDW. After
\end{abstract}

Correspondence to: Dr Min Tao, Department of Oncology, The First Affiliated Hospital of Soochow University, 188 Shizi Street, Suzhou, Jiangsu 215006, P.R. China

E-mail: taomin@suda.edu.cn

Dr Wen-Jie Wang, Department of Radio-Oncology, The Affiliated Suzhou Hospital of Nanjing Medical University, 16 Baita West Road, Suzhou, Jiangsu 215001, P.R. China

E-mail: doctor.wjwang@gmail.com

*Contributed equally

Key words: colorectal cancer, platelet, platelet-associated indicators, prognosis, overall survival the whole course of treatment (surgery combined with adjuvant chemotherapy), PLT, PCT and PDW were significantly decreased. Kaplan-Meier plots illustrated that patients with a post-/pre-treatment MPV ratio $<1$ had poorer overall survival (OS), whereas the post-/pre-treatment ratios for PLT, PCT and PDW did not correlate with patient outcome. Multivariate Cox regression analysis revealed that sex, tumor size and the post-/pre-treatment MPV ratio were prognostic factors for OS. Therefore, the present results may suggest MPV as a potential prognostic factor in resectable CRC.

\section{Introduction}

Colorectal cancer (CRC) is one of the most common cancer types globally (1). In the United States, New Zealand and France the incidence and mortality rates of CRC have exhibited a downward trend, resulting from early screening, improved therapy and preventive lifestyle choices (2). The primary preventive measure for $\mathrm{CRC}$ is a healthy lifestyle, including the maintenance of a healthy weight, exercise, reduction of meat and alcohol consumption, and avoidance of smoking (1). It is widely accepted that surgical resection is the principal therapy option at the early stages of CRC, which is defined as invasion and lymph node metastasis only occurring locally (3). In addition, adjuvant chemotherapy is an essential treatment for numerous patients with resectable CRC (4).

Platelets are involved in multiple mechanisms of cancer development and metastasis (5). Platelet-associated indicators assessed by routine blood examination include platelet count (PLT), mean platelet volume (MPV), platelet distribution width (PDW) and plateletcrit (PCT). MPV reflects the average size, stimulation and production rate of platelets. Pro-inflammatory and pro-thrombotic conditions are closely associated with MPV $(6,7)$. PCT is calculated as the MPV multiplied by the PLT. It has been identified that an elevated PCT is associated with coronary artery disease and an increased risk of venous thrombosis $(8,9)$. The PDW is one of 
the platelet volume indicators, reflecting the average change in platelet volume (10). Therefore, platelet-associated indicators may be used as inflammatory markers for CRC, in addition to their use in inflammatory, thromboembolic and cardiovascular diseases.

In the present study, the hypothesis that platelet-associated indices may provide useful prognostic information was investigated in patients with resectable CRC.

\section{Materials and methods}

Subjects and inclusion criteria. The present study was conducted as a retrospective investigation of patients with resectable CRC that had been referred to the First Affiliated Hospital of Soochow University (Jiangsu, China) between June 2006 and July 2016. The Medical Ethics Committees of the First Affiliated Hospital of Soochow University granted approval for the present study, and written informed consent to participate was obtained from patients at the beginning of the study. The clinical and pathological records of all participating patients were reviewed periodically.

In total, 153 patients with resectable CRC were recruited. All cases were confirmed by surgery and pathology. All patients originally received six cycles of XELOX treatment, a standard adjuvant chemotherapy including oxaliplatin administered intravenously at a dose of $130 \mathrm{mg} / \mathrm{m}^{2}$ on day 1 , and capecitabine $\left(850-1,250 \mathrm{mg} / \mathrm{m}^{2}\right)$ twice a day for 2 weeks, followed by a 1 -week rest. Patient characteristics are detailed in Table I. The median age of the patients was 56 years (range, 27-85 years); 70 patients were male and 83 were female. Cancer stage was determined using the Tumor-Node-Metastasis (TNM) classification according to the recommendations of the American Joint Committee on Cancer (AJCC) (11). Prognostic analyses were performed in regard to overall survival (OS).

Blood samples. Pre-surgery blood samples were collected up to 1 week before surgery. Post-surgery blood samples were regarded as pre-chemotherapy samples and were collected 3 weeks after surgery. Post-chemotherapy samples were collected after 3 months of standard chemotherapy in order to eliminate the impact of treatment on platelet-associated indicators. Peripheral venous blood $(5-7 \mathrm{ml})$ was collected into sterile EDTA tubes. All blood samples were fasted and obtained between 6:30 and 7:30 a.m. in order to standardize the known impact of circulating hormones (circadian rhythm) on the number and subtype distribution of various white blood cell indices (12). Hematological parameters were analyzed up to $30 \mathrm{~min}$ after collection, using a hematology analyzer (Sysmex XE-2100; Sysmex Corporation). PLT, PCT, MPV and PDW blood levels are recorded in Table I. The patients were divided into two groups according to the median value of PLT, PCT, MPV or PDW. The post-/pre-treatment ratios were defined as the rate of pre- and post-treatment blood parameter values.

Computed tomography (CT) evaluation. CT scans were performed every 2 months for response assessment and evaluated according to the criteria of the Response Evaluation Criteria in Solid Tumors 1.1 (13).
Follow-up. Patient survival time was recorded from the date of chemotherapy until death or the last clinical evaluation. Prognostic analyses were performed in relation to patient OS time. OS was defined as the time from diagnosis to that of death from any cause.

Statistical analysis. All statistical analyses were performed using SPSS 19.0 software (IBM Corp.). For analysis of survival data, Kaplan-Meier curves were constructed and statistical analysis was performed using the log-rank test. The associations between blood-parameter status and clinicopathological features were determined using the $\chi^{2}$ test. The associations between alterations in the status of platelet-associated indicators and surgery or chemotherapy were assessed using a Student's t-test. The multivariate logistic regression model was employed to identify the independent risk factors associated with resectable CRC. $\mathrm{P}<0.05$ was considered to indicate a statistically significant difference.

\section{Results}

Pre-treatment PLT, PCT, MPV and PDW values do not correlate with the outcome of patients with resectable CRC. Kaplan-Meier plots were used to determine the effects of pre-treatment PLT, PCT, MPV and PDW status on OS (Fig. 1). The patients were divided into two groups according to the median value of PLT (low PLT, $\leq 211.575 \times 10^{9} / 1$, or high PLT, $>211.575 \times 10^{9} / 1$ ), PCT (low PCT, $\leq 0.2251 / 1$, or high PCT, $>0.225$ 1/1), MPV (low MPV, $\leq 10.400 \mathrm{fl}$, or high MPV, $>10.400 \mathrm{fl}$ ) or PDW (low PDW, $\leq 15.485 \%$, or high PDW, $>15.485 \%$ ). The mean OS of the high PLT group was 63 months (range, 53.392-72.608), while that of the low PLT group was 56 months (range, 49.312-62.688; $\mathrm{P}=0.956$ ). The mean OS was 58 months (range, 52.731-63.269) in the high PCT group and 64 months (range, 51.806-76.194) in the low PCT group $(\mathrm{P}=0.496)$. The mean OS of the high MPV group was 58 months (range, 53.728-62.272), while that of the low MPV group was 68 months (range, 46.025-89.975; $\mathrm{P}=0.107$ ). The mean OS was 52 months (range, 44.465-59.535) in the high PDW group and 63 months (range, 58.623-67.377) in the low PDW group $(\mathrm{P}=0.300)$. These results demonstrated that pre-treatment levels of PLT, PCT, MPV or PDW had no effects on the OS of patients with resectable CRC.

Surgery significantly decreases MPV, but has no impact on PLT, PCT or PDW. The effects of surgery on the values for PLT, PCT, MPV and PDW are presented in Fig. 2. The median PLT value was $216.000 \times 10^{9} / 1$ [95\% confidence interval (CI),

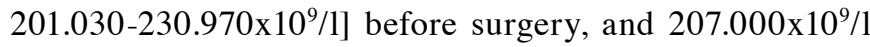
(95\% CI, 194.210-219.800×10\%/1) after surgery $(\mathrm{P}=0.278)$. The median PCT value was $0.2001 / 1$ (95\% CI, 0.190-0.220 1/1) before surgery, and $0.190 \mathrm{~L} / \mathrm{L}$ (95\% CI, 0.170-0.220 $1 / 1)$ after surgery $(\mathrm{P}=0.407)$. The median MPV value was $10.000 \mathrm{fl}(95 \% \mathrm{CI}, 9.680-10.400 \mathrm{fl})$ before surgery, and $9.900 \mathrm{fl}$ (95\% CI, 9.300-10.198 fl) after surgery $(\mathrm{P}<0.001)$. The median PDW value was $15.600 \%$ (95\% CI, 15.040-15.900\%) before surgery, and $15.300 \%$ (95\% CI, 14.760-15.700\%) after surgery $(\mathrm{P}=0.344)$. Therefore, surgery significantly decreased the MPV value, but had no significant impact on the PLT, PCT and PDW values of patients with resectable CRC. 


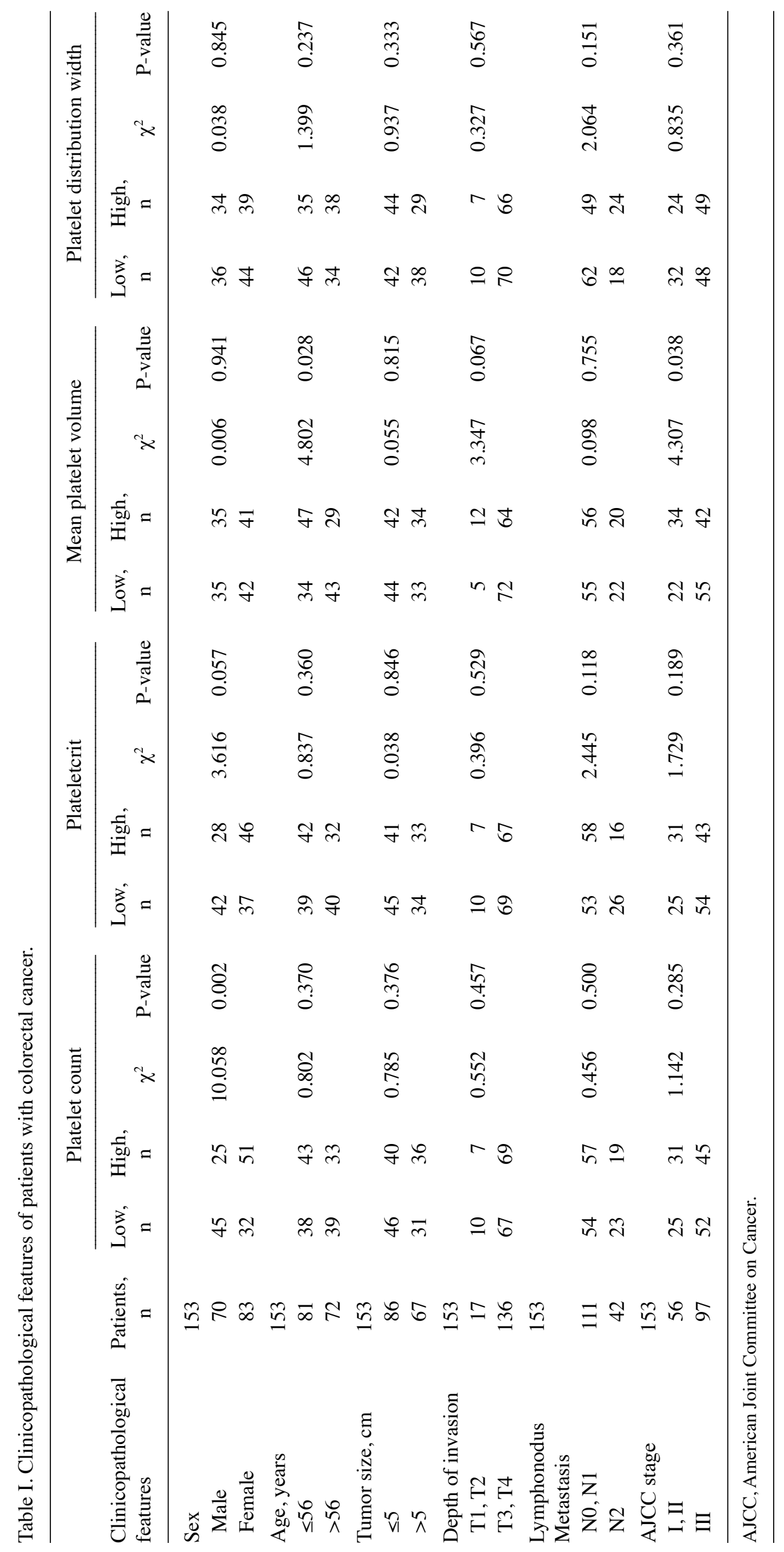


A

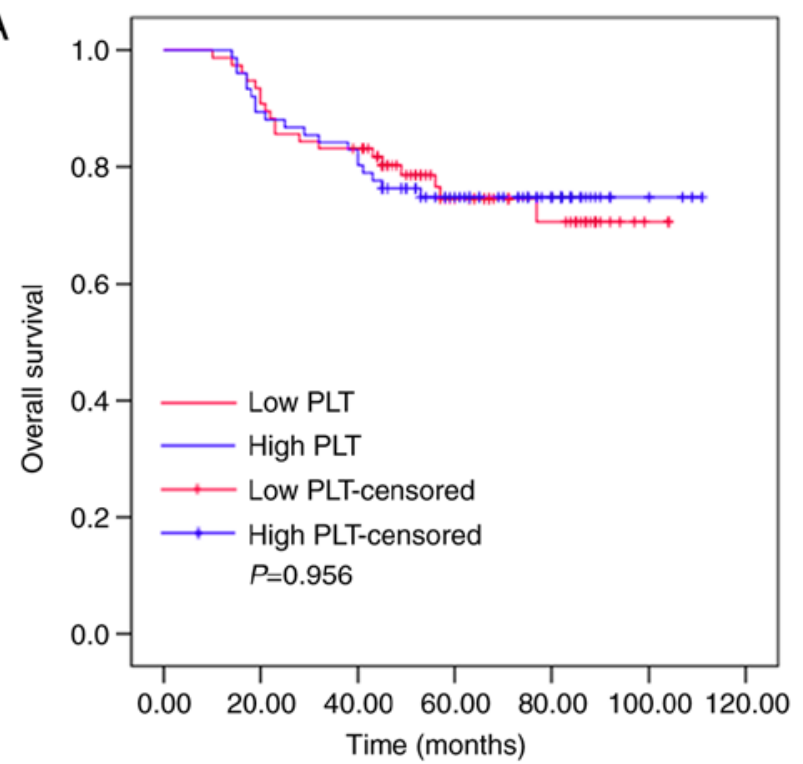

C

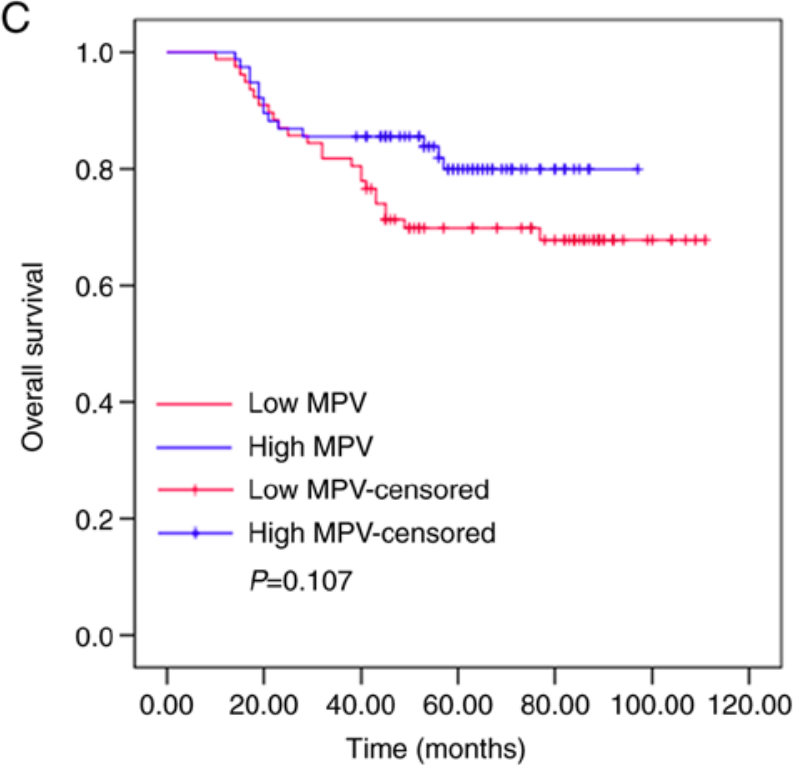

B

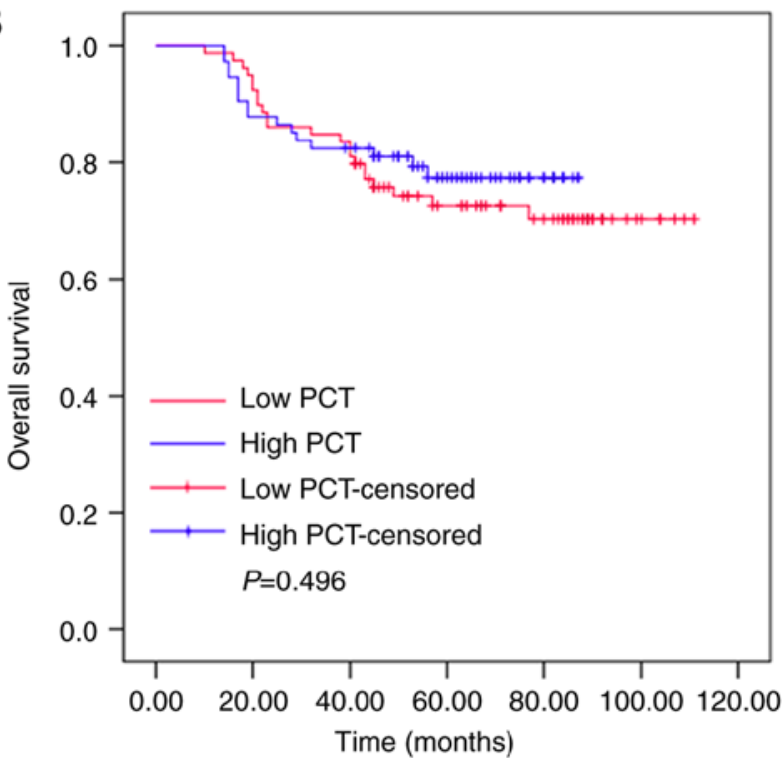

D

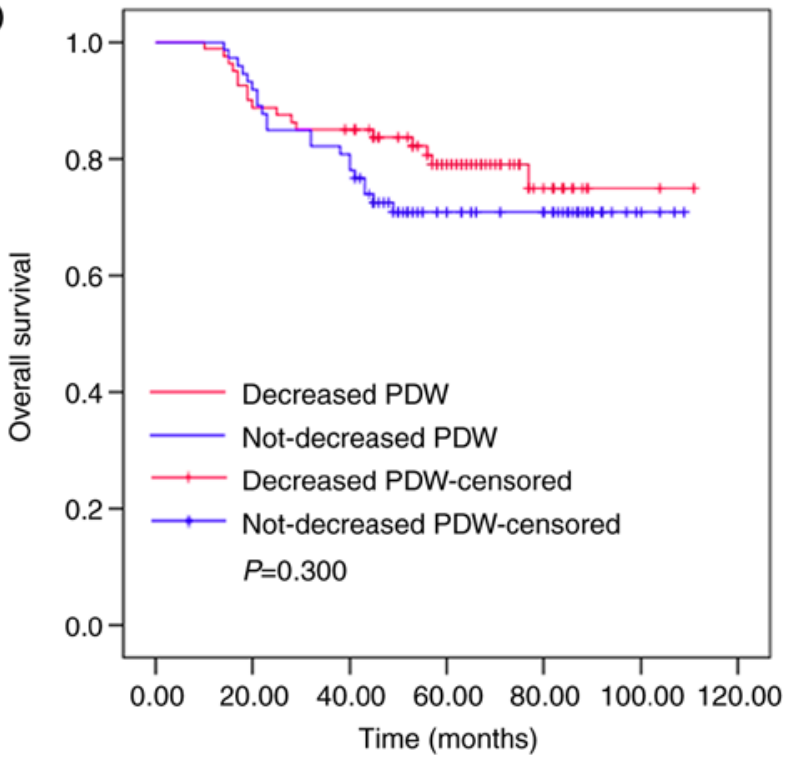

Figure 1. Pre-treatment PLT, PCT, MPV and PDW values are not associated with the outcome of patients with resectable colorectal cancer. The OS according to (A) PLT, (B) PCT, (C) MPV and (D) PDW. OS, overall survival; PLT, platelet count; PCT, plateletcrit; MPV, mean platelet volume; PDW, platelet distribution width.

Adjuvant chemotherapy significantly decreases PLT and PCT, and increases MPV, but has no effect on PDW. The effects of adjuvant chemotherapy on the values for PLT, PCT, MPV and PDW are shown in Fig. 3. The median PLT value was

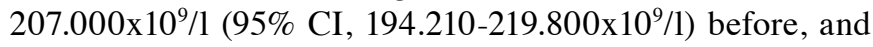
$157.000 \times 10^{9} / 1(95 \%$ CI, 149.160-164.840x10\%/l) after adjuvant chemotherapy $(\mathrm{P}<0.001)$. The median $\mathrm{PCT}$ value was 0.190 1/1 (95\% CI, 0.170-0.220 1/1) before, and 0.170 1/1 (95\% CI, $0.160-0.1801 / 1)$ following adjuvant chemotherapy $(\mathrm{P}<0.001)$. The median MPV value was $9.900 \mathrm{fl}(95 \% \mathrm{CI}, 9.300-10.198 \mathrm{fl})$ before, and $10.500 \mathrm{fl}$ (95\% CI, 10.200-10.800 fl) following adjuvant chemotherapy $(\mathrm{P}<0.001)$. The median PDW value was $15.300 \%$ (95\% CI, 14.760-15.700\%) before adjuvant chemotherapy, and $14.300 \%$ (95\% CI, 13.200-15.000\%) after adjuvant chemotherapy $(\mathrm{P}=0.093)$. Adjuvant chemotherapy significantly decreased the PLT and PCT values, but increased the MPV value of patients with resectable CRC. There was no significant impact on the PDW value.

A whole course of treatment significantly decreases PLT, PCT and PDW, but has no effect on MPV. The impact of the whole course of treatment (surgery and adjuvant chemotherapy) on the values for PLT, PCT, MPV and PDW are displayed in Fig. 4. The median PLT value was $216.000 \times 10^{9} / 1$ (95\% CI,

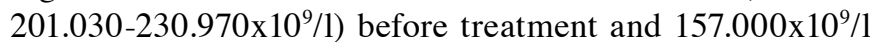
(95\% CI, 149.160-164.840x10 $/ 1)$ after treatment $(\mathrm{P}<0.001)$. The PCT median value was 0.200 1/l (95\% CI, 0.190-0.220 1/l) before treatment and 0.170 1/1 (95\% CI, 0.160-0.180 1/1) after treatment $(\mathrm{P}<0.001)$. The median MPV value was $10.000 \mathrm{fl}$ $(95 \% \mathrm{CI}, 9.680-10.400 \mathrm{fl})$ before treatment and $10.500 \mathrm{fl}$ $(95 \%$ CI, 10.200-10.800 fl) after treatment $(\mathrm{P}=0.322)$. The median PDW value was $15.600 \%$ (95\% CI, $15.040-15.900 \%$ ) 

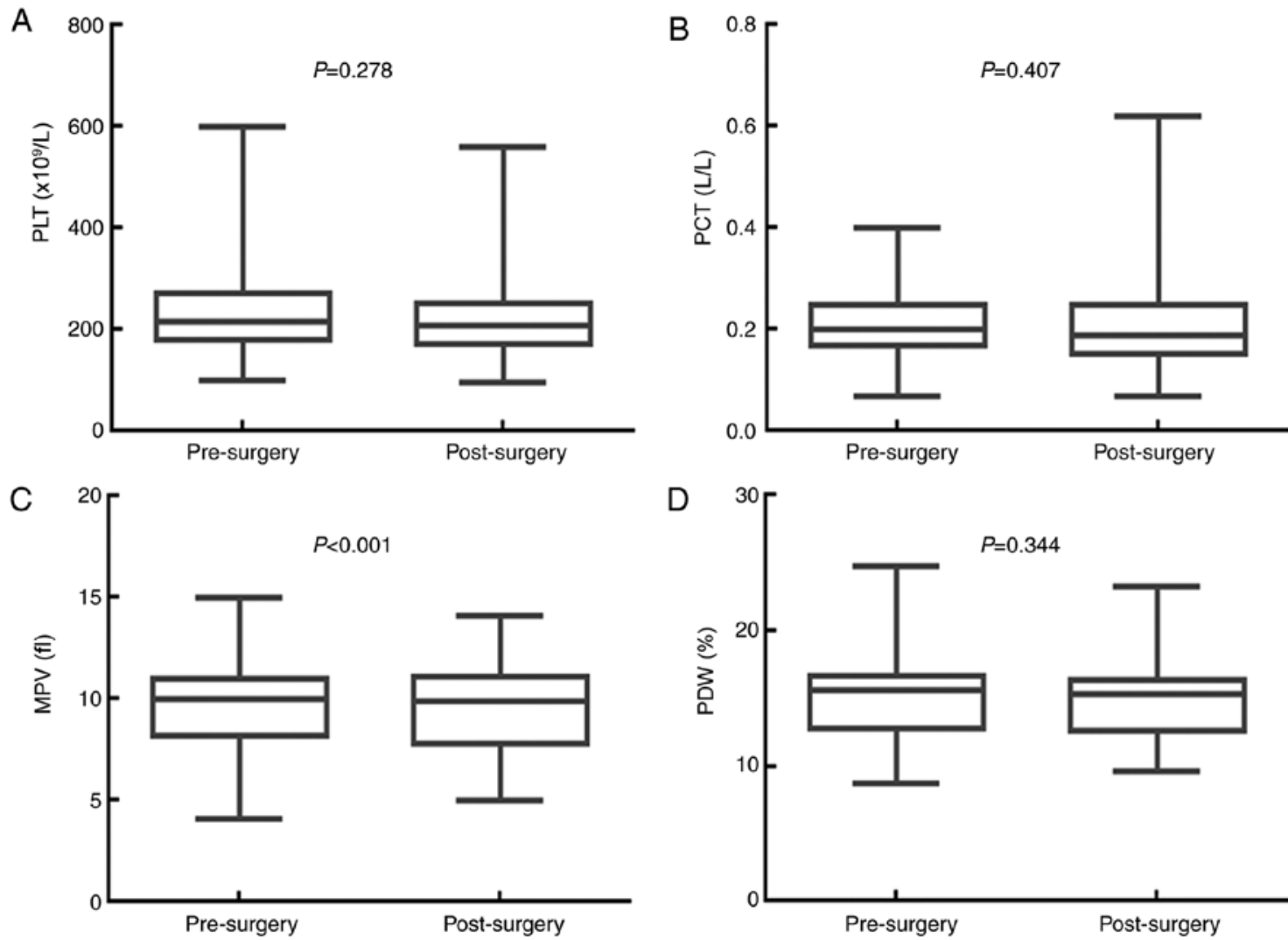

Figure 2. Surgery significantly decreases the MPV value in patients with resectable colorectal cancer. Surgery had no influence on (A) PLT or (B) PCT. (C) Surgery significantly decreased the MPV of patients with colorectal cancer. (D) Surgery had no influence on the PDW. PLT, platelet count; PCT, plateletcrit; MPV, mean platelet volume; PDW, platelet distribution width.
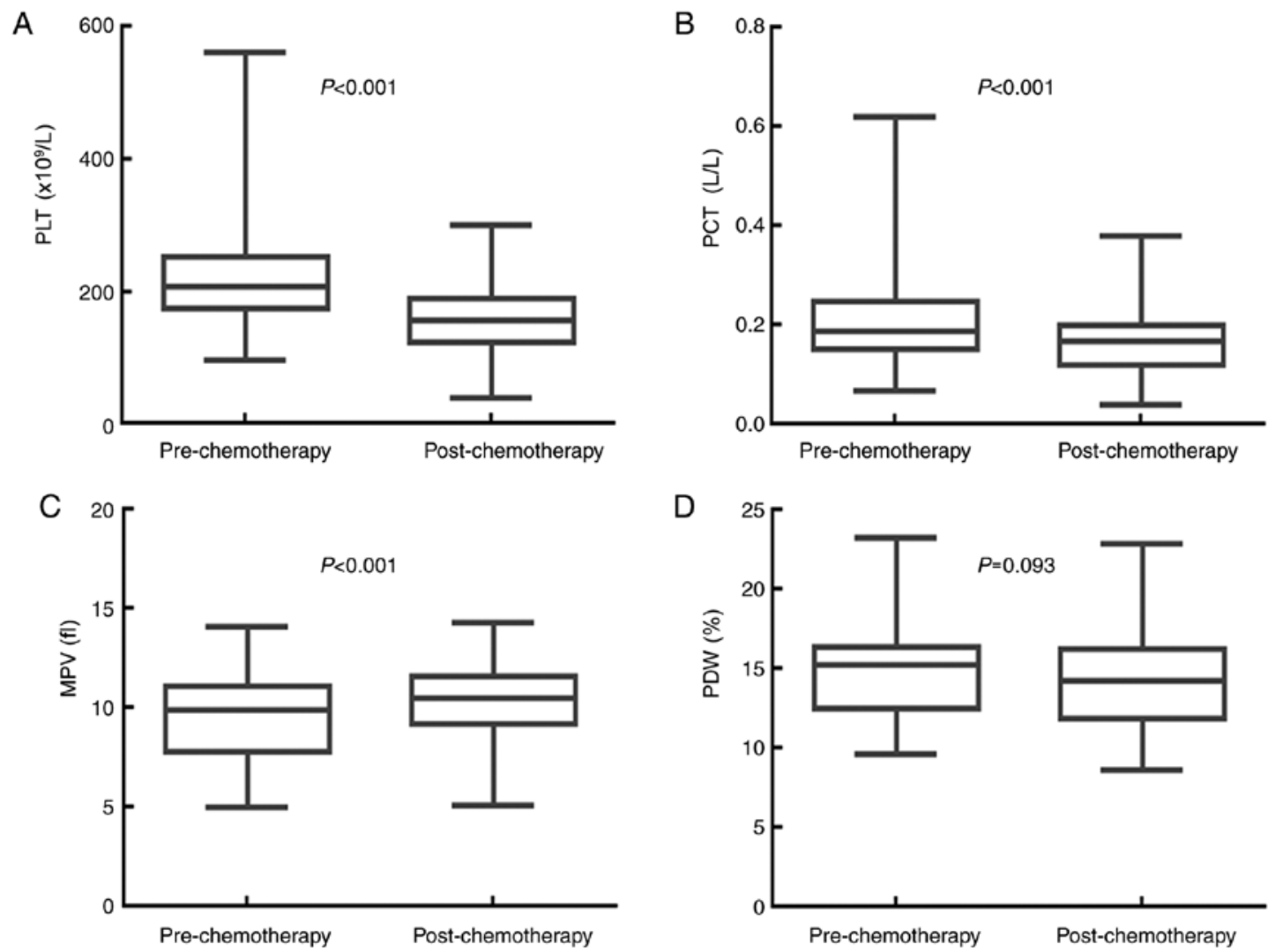

Figure 3. Adjuvant chemotherapy significantly decreases the values of PLT and PCT, and increases MPV in patients with resectable CRC. Chemotherapy significantly decreased the (A) PLT and (B) PCT, and significantly increased the (C) MPV of patients with CRC. (D) Chemotherapy had no influence on the PDW of patients with CRC. CRC, colorectal cancer; PLT, platelet count; PCT, plateletcrit; MPV, mean platelet volume; PDW, platelet distribution width. 
A

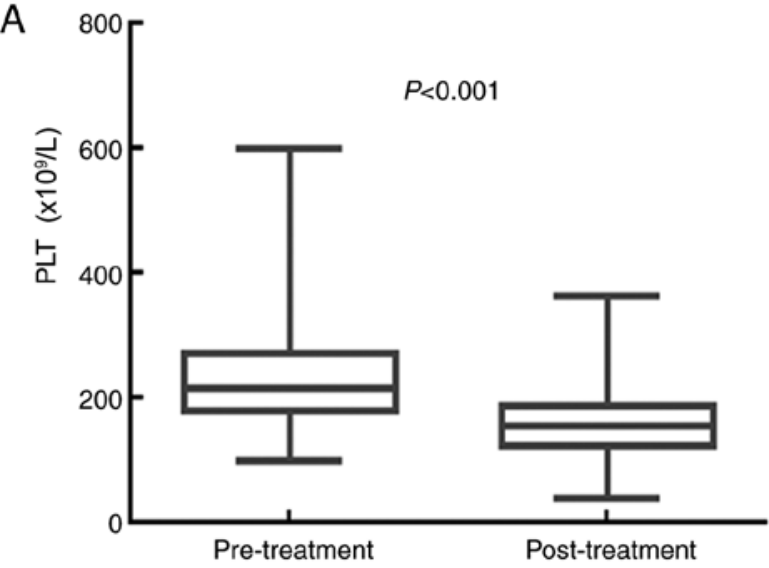

C

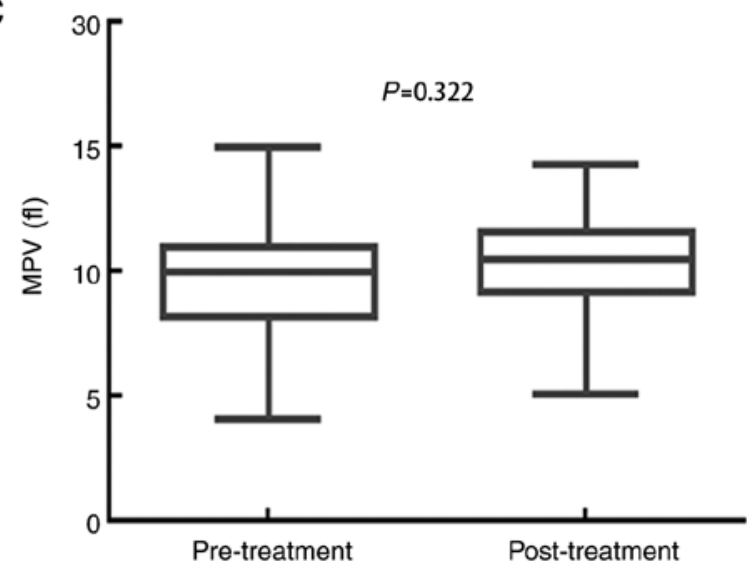

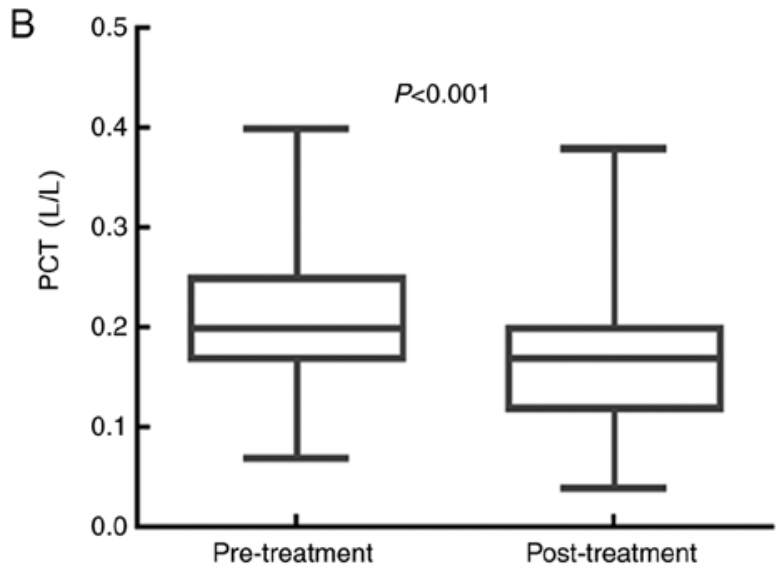

D

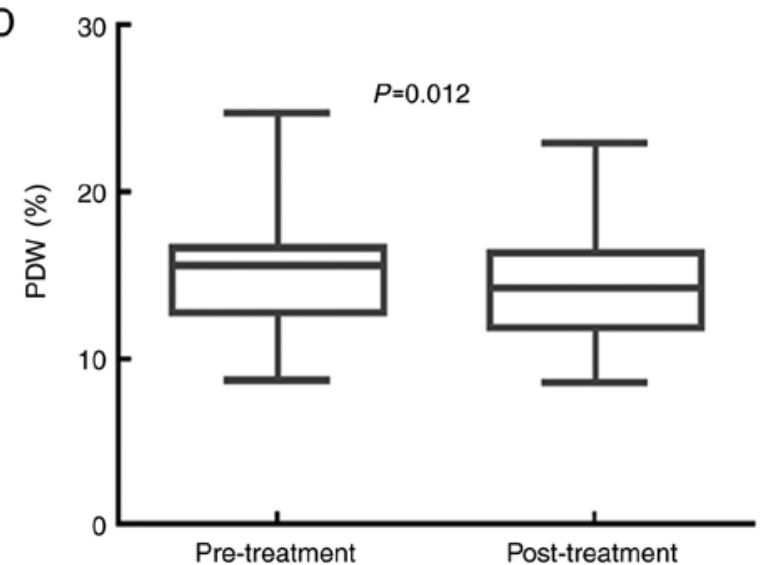

Figure 4. PLT, PCT and PDW values are significantly decreased in patients with CRC following a whole course of treatment. A whole course of treatment (surgery combined with adjuvant chemotherapy) significantly decreased the (A) PLT and (B) PCT of patients with CRC. Combined treatment had no influence on the (C) MPV, but significantly decreased the (D) PDW of patients with CRC. CRC, colorectal cancer; PLT, platelet count; PCT, plateletcrit; MPV, mean platelet volume; PDW, platelet distribution width.

before treatment, and $14.300 \%$ (95\% CI, 13.200-15.000\%) after treatment $(\mathrm{P}=0.012)$. Treatment with surgery and adjuvant chemotherapy significantly decreased the PLT, PCT and PDW values, but had no obvious effect on the MPV value of patients with resectable CRC.

Changes in MPV following a whole course of treatment predict the outcomes of patients with resectable CRC. Kaplan-Meier plots were used to determine the effect of changes in PLT, PCT, MPV and PDW status on OS (Fig. 5). The mean OS of patients whose MPV decreased following a whole course of treatment (surgery and adjuvant chemotherapy) was 75 months (range, 65.918-85.211), while that of patients whose MPV did not decrease was 97 months (range, 90.599-103.210). Significant differences were identified for OS between these two groups $(\mathrm{P}=0.005)$. The mean OS of patients whose PLT level decreased following a whole course of treatment was 92 months (range, 85.875-97.804), while that of patients whose PLT did not decrease was 62 months (range, 46.703-77.742; $\mathrm{P}=0.096$ ). The mean OS of patients whose PCT level decreased following a whole course of treatment was 92 months (range, 85.409-98.433), while that of the non-decreased group was 78 months (rang, 66.979-90.514; $\mathrm{P}=0.192$ ). The mean OS of patients whose PDW level decreased following a whole course of treatment was 77 months (range, 71.466-83.015), while those where PDW did not decrease was 88 months (range, 79.668-97.597; $\mathrm{P}=0.744$ ). Thus, the patients whose MPV level increased following surgery and adjuvant chemotherapy had increased survival ratios. However, changes in PLT, PCT and PDW had no significant effect on OS.

Univariate and multivariate analyses of prognostic factors in patients with resectable CRC. Univariate analyses demonstrated that sex [female; hazard ratio (HR), 2.911; 95\% confidence interval $(\mathrm{CI}), 1.413-5.998 ; \mathrm{P}=0.004)$, tumor size $(>5 \mathrm{~cm}$; HR 2.613; 95\% CI 1.351-5.054; $\mathrm{P}=0.004)$, lymphonodus metastasis (N2; HR 2.197; 95\% CI 1.153-4.187; $\mathrm{P}=0.017)$, AJCC stage (III; HR 2.408; 95\% CI 1.103-5.254; $\mathrm{P}=0.027$ ) and decreased post/pre-treatment MPV ratio (HR 2.430; 95\% CI 1.274-4.636; $\mathrm{P}=0.007)$ were significant risk factors for poor prognosis (Table II). In multivariate analysis, sex (female; HR 3.236; 95\% CI 1.553-6.745; $\mathrm{P}=0.002)$, tumor size ( $>5 \mathrm{~cm}$; HR 2.058; 95\% CI 1.007-4.203; $\mathrm{P}=0.048)$ and decreased post/pre-treatment MPV ratio (HR 2.621; 95\% CI 1.329-5.131; $\mathrm{P}=0.005$ ) were found to be independently associated with poor survival.

\section{Discussion}

Platelets, derived from megakaryocytes, have a crucial role in a variety of physiological and pathological processes, 

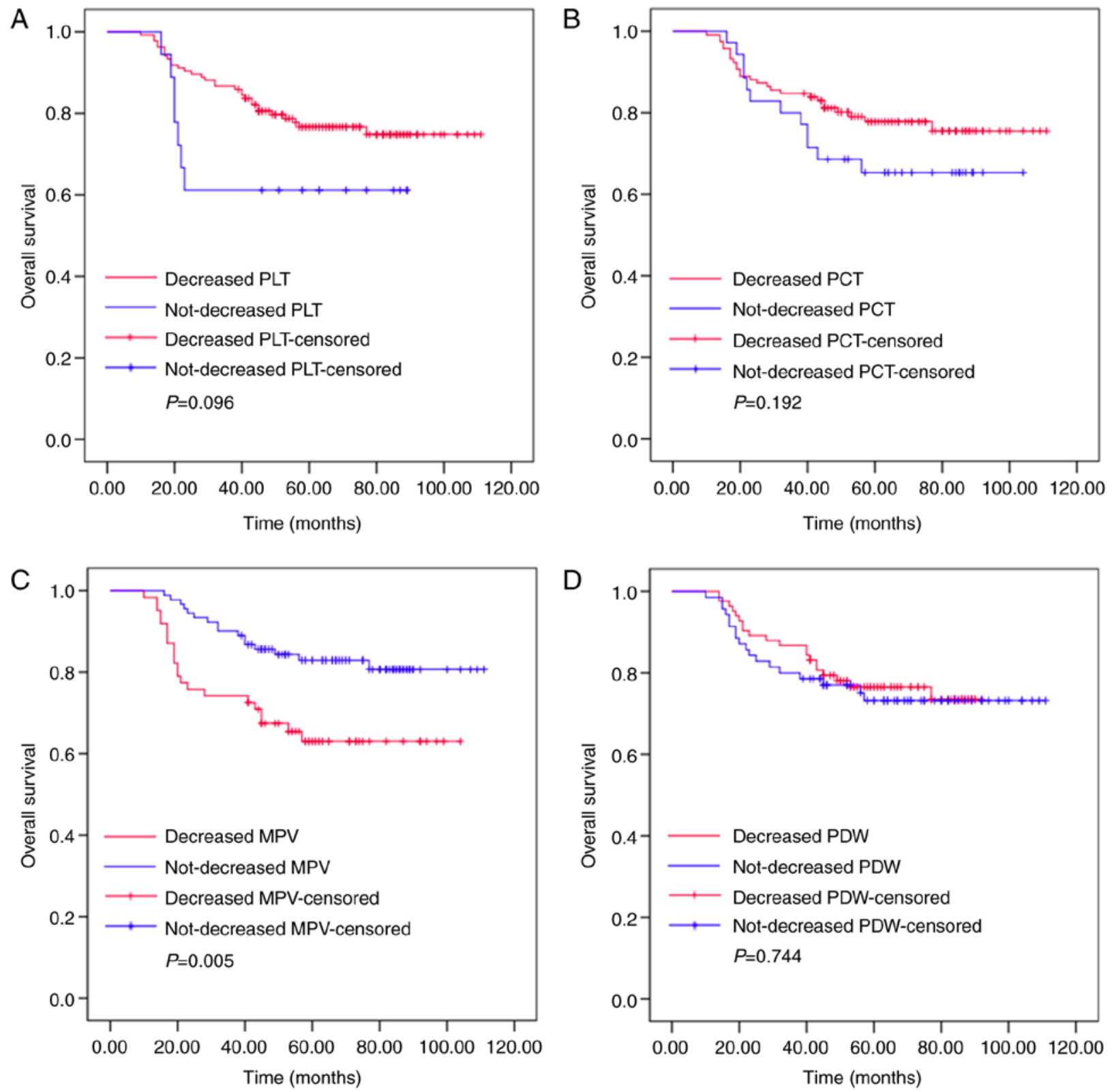

Figure 5. Alterations in MPV following a whole course of treatment predict the outcomes of patients with resectable CRC. The OS of patients with CRC according to changes in (A) PLT, (B) PCT, (C) MPV and (D) PDW. CRC, colorectal cancer; PLT, platelet count; PCT, plateletcrit; MPV, mean platelet volume; PDW, platelet distribution width.

including hemostasis, wound healing, inflammatory responses and thrombosis. Previous studies have confirmed that a high PLT, which is associated with tumorigenesis, metastasis and angiogenesis, is a common phenomenon in patients with solid cancer types $(14,15)$. Meta-analyses have suggested that a high PLT may have diagnostic and prognostic value in various types of cancer, including CRC $(16,17)$. Platelet activation is involved in progressive tumor growth and metastasis via the release of cytokines, chemokines and the expression of numerous adhesion receptors $(18,19)$. Cancer cell-derived interleukin (IL-)6 is a trigger for high, tumor-induced PLT in several types of cancer $(20,21)$. By protecting circulating tumor cells from immune-surveillance, platelets contribute to metastasis (14). In addition, the obstructions formed by platelets and tumor cells in the microcirculation facilitate the extravasation of tumor cells to metastatic sites. In the present study, surgery combined with adjuvant chemotherapy significantly decreased the PLT of patients with CRC. Chemotherapy was likely the primary cause of this downregulation, since surgery had no effect on PLT status. However, the post/pre-treatment ratio of PLT, which was based on changes in individual PLTs, had no apparent association with OS.

Platelet-associated indicators are closely associated with the activation and function of platelets. MPV reflects early platelet activation $(6-10,14-22)$ and PCT, obtained by multiplying the PLT and the MPV, reflects the percentage of the blood volume occupied by platelets. The PDW has been one of the most commonly used markers to indicate platelet activity. According to previous studies, there may be a correlation between platelet-associated indicators and the prognosis of patients with cancer $(23,24)$. The PCT differs depending on the stage, histological type and metastatic status of various 
Table II. Univariate and multivariate logistic regression analysis of risk factors for resectable colorectal cancer.

\begin{tabular}{|c|c|c|c|c|}
\hline \multirow[b]{3}{*}{ Risk factors } & \multicolumn{4}{|c|}{ Overall survival } \\
\hline & \multicolumn{2}{|c|}{ Univariate analysis } & \multicolumn{2}{|c|}{ Multivariate analysis } \\
\hline & OR $(95 \% \mathrm{CI})$ & P-value & OR $(95 \% \mathrm{CI})$ & P-value \\
\hline \multicolumn{5}{|l|}{ Sex } \\
\hline Female or male & $2.911(1.413-5.998)$ & 0.004 & $3.236(1.553-6.745)$ & 0.002 \\
\hline \multicolumn{5}{|l|}{ Age } \\
\hline$>56$ or $\leq 56$ years & $1.003(0.974-1.034)$ & 0.825 & - & - \\
\hline \multicolumn{5}{|l|}{ Tumor size, $\mathrm{cm}$} \\
\hline$>5$ or $\leq 5$ & $2.613(1.351-5.054)$ & 0.004 & $2.058(1.007-4.203)$ & 0.048 \\
\hline \multicolumn{5}{|l|}{ Depth of invasion } \\
\hline $\mathrm{T} 3-4$ or $\mathrm{T} 1-2$ & $2.591(0.624-10.77)$ & 0.190 & - & - \\
\hline \multicolumn{5}{|l|}{ Lymphonodus, metastasis } \\
\hline $\mathrm{N} 2$ or $\mathrm{N} 0-1$ & $2.197(1.153-4.187)$ & 0.017 & $2.070(0.967-4.429)$ & 0.061 \\
\hline \multicolumn{5}{|l|}{ AJCC stage } \\
\hline III or I-II & $2.408(1.103-5.254)$ & 0.027 & $1.440(0.588-3.529)$ & 0.425 \\
\hline \multicolumn{5}{|l|}{ Pre-treatment PLT } \\
\hline$\leq 211.575$ or $>211.575 \times 10^{9} / 1$ & $1.018(0.539-1.924)$ & 0.956 & - & - \\
\hline \multicolumn{5}{|l|}{ Pre-treatment PCT } \\
\hline$\leq 0.225$ or $>0.2251 / 1$ & $1.250(0.656-2.383)$ & 0.498 & - & - \\
\hline \multicolumn{5}{|l|}{ Pre-treatment MPV } \\
\hline$\leq 10.400$ or $>10.400 \mathrm{fl}$ & $1.709(0.883-3.309)$ & 0.112 & - & - \\
\hline \multicolumn{5}{|l|}{ Pre-treatment PDW } \\
\hline$\leq 15.485$ or $>15.485 \%$ & $0.714(0.376-1.356)$ & 0.304 & - & - \\
\hline \multicolumn{5}{|l|}{ Post-/pre-treatment PLT ratio } \\
\hline$<1$ or $\geq 1$ & $0.506(0.222-1.150)$ & 0.104 & - & - \\
\hline \multicolumn{5}{|l|}{ Post-/pre-treatment PCT ratio } \\
\hline$<1$ or $\geq 1$ & $0.638(0.322-1.264)$ & 0.197 & - & - \\
\hline \multicolumn{5}{|l|}{ Post-/pre-treatment MPV ratio } \\
\hline$<1$ or $\geq 1$ & $2.430(1.274-4.636)$ & 0.007 & $2.612(1.329-5.131)$ & 0.005 \\
\hline \multicolumn{5}{|l|}{ Post-/pre-treatment PDW ratio } \\
\hline$<1$ or $\geq 1$ & $0.893(0.472-1.688)$ & 0.727 & - & - \\
\hline
\end{tabular}

OR, odds ratio; CI confidence interval; PLT, platelet count; PCT, plateletcrit; MPV, mean platelet volume; PDW, platelet distribution width; AJCC, American Joint Committee on Cancer.

types of cancer. For instance, the PCT is higher in patients with epithelial ovarian or endometrial cancer compared with that in healthy controls $(25,26)$. PCT was also reported to be positively correlated with common causes of mortality, including venous thrombosis and tumor metastasis, in patients with CRC $(8,27,28)$. Although surgery and adjuvant chemotherapy significantly reduced PCT in the present study, the post/pre-treatment ratio of PCT was not associated with OS.

The implication of the MPV is controversial at present. Certain studies have indicated that MPV in patients with cancer was higher compared with that in normal controls $(29,30)$. In addition, other studies have reported a positive correlation between high MPV and advanced cancer stage $(24,31)$. However, several studies have indicated the opposite, stating that a low MPV was associated with unfavorable prognoses in non-small cell lung cancer (NSCLC) $(32,33)$. Kumagai et al (33) determined that in patients with resectable NSCLC, an MPV of $<8.50 \mathrm{fl}$ was an independent predictive indicator for shorter disease-free survival and OS. In addition, Tuncel et al (34) demonstrated that the MPV was significantly higher in patients with metastatic CRC (mCRC) compared with that in patients with non-mCRC, and that the benefits of bevacizumab on progression-free survival were significantly greater in patients with low MPV, compared with those with high MPV status. Furthermore, in a study by Li et al (35), patients with a low MPV ( $\leq 8.6 \mathrm{fl})$ had a significantly improved OS compared with those with a high MPV (>8.6 fl). Multivariate analysis revealed that high MPV 
was an independent risk factor affecting OS in patients with resectable CRC. In the present study, surgery increased MPV, while chemotherapy decreased it, leading to an MPV that was unchanged (compared with baseline) following treatment with both surgery and adjuvant chemotherapy. Furthermore, based on individual MPV values, subjects whose MPV's were not decreased had better outcomes. Univariate and multivariate analyses demonstrated that a decreased post/pre-treatment MPV ratio was a significant and independent risk factor associated with poor prognosis. Therefore, an unchanged MPV following treatment may indicate a favorable prognostic index in resectable CRC.

The association between decreased MPV and poor prognosis may be due to a number of variables. The key to explaining this decrease may be the association between blood coagulation and cancer in general. A study has demonstrated that tumor necrosis factor- $\alpha$, IL- $1 \beta$, vascular endothelial growth factor and basic fibroblast growth factor released from various cells stimulated the formation of vascular endothelial thrombi, and enhanced the destruction of larger-sized platelets, resulting in a decreased MPV in the circulating platelets (36). Another study suggested that increased thrombotic activity in patients with metastatic colon cancer led to a decrease in the MPV, which was in most cases considered to correlate with poor survival (37).

PDW, another platelet index, has been reported to be a diagnostic and prognostic factor for cancer (38). Oncel et al (10) suggested that the PDW was significantly higher in patients with lung cancer compared with that in the control group. Ma et al (25) identified that the PDW was higher in the epithelial ovarian cancer group compared with that in the benign tumor and healthy control groups. Hirahara et al (39) focused on PDW and cancer-specific survival in patients with esophageal cancer. Due to a small proportion of patients with an elevated PDW, it was not possible to assess the statistical association between PDW and prognostic factors. In a recent retrospective study by Song et al (40), univariate analysis revealed that an elevated PDW was an independent risk factor for recurrence-free survival and OS in patients with non-mCRC. In the present study, it was identified that surgery combined with adjuvant chemotherapy significantly decreased PDW. However, the post/pre-treatment ratio of PDW had no significant impact on OS.

In conclusion, the present study indicated that an increased MPV following a whole course of treatment was associated with improved OS in patients with resectable CRC, and that a low post-/pre-treatment MPV ratio was an independent negative prognostic factor for OS. Of note, the present study had certain limitations. It was a retrospective study with a small sample size, involving only 153 patients. Patients with systemic diseases, including hypertension, diabetes, rheumatic disease and infection were excluded in order to eliminate the impact of these conditions on platelet-associated indicators. In spite of the small sample size, the results of the present study were representative to a certain extent. A multi-center study with a larger sample population will be performed in the future.

\section{Acknowledgements}

Not applicable.

\section{Funding}

The present study was supported by the National Natural Science Foundation of China (grant nos. 81472296, 816 02091, 81402176, 81402093, 81272542 and 81200369), the Six Major Talent Peak Project of Jiangsu Province (grant no. 2015-WSN-022), the Project of Invigorating Health Care through Science, Technology and Education, Jiangsu Provincial Medical Youth Talent (grant no. QNRC2016709), the Project of Jiangsu Provincial Commission of Health and Family Planning (grant no. H201518), the Science and Education for Health Foundation of Suzhou for Youth (grant no. kjxw2015003), and the Science and Technology Project Foundation of Suzhou (grant nos. SYS201464 and SYS201504).

\section{Availability of data and materials}

The datasets used and/or analyzed during the current study are available from the corresponding author on reasonable request.

\section{Authors' contributions}

MT and WJW designed the study. WQ, JW, FRG and MYW collected the data. MDX, LL and WL carried out the statistical analysis. XXG interpreted and checked the data. WQ and XXG wrote the paper. WQ and MDX were responsible for the revision of the manuscript. All authors read and approved the final manuscript.

\section{Ethics approval and consent to participate}

The Medical Ethics Committees of the First Affiliated Hospital of Soochow University granted approval for the present study. Written informed consent was obtained from all patients.

\section{Patient consent for publication}

All patients provided consent for the publication of their data and associated images.

\section{Competing interests}

The authors declare that they have no competing interests.

\section{References}

1. Torre LA, Bray F, Siegel RL, Ferlay J, Lortet-Tieulent J and Jemal A: Global cancer statistics, 2012. CA Cancer J Clin 65: 87-108, 2015.

2. Torre LA, Siegel RL, Ward EM and Jemal A: Global cancer incidence and mortality rates and trends-an update. Cancer Epidemiol Biomarkers Prev 25: 16-27, 2016.

3. Punt CJ, Koopman M and Vermeulen L: From tumour heterogeneity to advances in precision treatment of colorectal cancer. Nat Rev Clin Oncol 14: 235-246, 2017.

4. Du C, Huang D, Peng Y, Yao Y, Zhao Y, Yang Y, Wang H, Cao L, Zhu WG and Gu J: 5-Fluorouracil targets histone acetyltransferases p300/CBP in the treatment of colorectal cancer. Cancer Lett 400: 183-193, 2017.

5. Ho-Tin-Noé B, Goerge T, Cifuni SM, Duerschmied D and Wagner DD: Platelet granule secretion continuously prevents intratumor hemorrhage. Cancer Res 68: 6851-6858, 2008. 
6. Colkesen $\mathrm{Y}$ and Muderrisoglu $\mathrm{H}$ : The role of mean platelet volume in predicting thrombotic events. Clin Chem Lab Med 50: 631-634, 2012

7. Lian L, Xia YY, Zhou C, Shen XM, Li XL, Han SG, Zheng Y, Gong FR, Tao $\mathrm{M}$ and $\mathrm{Li} \mathrm{W}$ : Mean platelet volume predicts chemotherapy response and prognosis in patients with unresectable gastric cancer. Oncol Lett 10: 3419-3424, 2015.

8. Vázquez-Santiago M, Vilalta N,Ziyatdinov A, Cuevas B, Macho R Pujol-Moix N, Carrasco M, Mateo J, Fontcuberta J, Soria JM and Souto JC: Platelet count and plateletcrit are associated with an increased risk of venous thrombosis in females. Results from the RETROVE study. Thromb Res 157: 162-164, 2017.

9. Ifran A, Hasimi A, Kaptan K, Nevruz O, Beyan C and Erbil K Evaluation of platelet parameters in healthy apheresis donors using the ADVIA 120. Transfus Apher Sci 33: 87-90, 2005.

10. Oncel M, Kiyici A, Sunam GS, Sahin E and Adam B Evaluation of platelet indices in lung cancer patients. Asian Pac J Cancer Prev 16: 7599-7602, 2015.

11. Kim KH, Yang SS, Yoon YS, Lim SB, Yu CS and Kim JC: Validation of the seventh edition of the American Join Committee on Cancer tumor-node-metastasis (AJCC TNM) staging in patients with stage II and stage III colorectal carcinoma: Analysis of 2,511 cases from a medical centre in Korea. Colorectal Dis 13: e220-e226, 2011.

12. Dimitrov S, Benedict C, Heutling D, Westermann J, Born J and Lange T: Cortisol and epinephrine control opposing circadian rhythms in T cell subsets. Blood 113: 5134-5143, 2009.

13. Keil S, Barabasch A, Dirrichs T, Bruners P, Hansen NL, Bieling HB, Brümmendorf TH and Kuhl CK: Target lesion selection: An important factor causing variability of response classification in the response evaluation criteria for solid tumors 1.1. Invest Radiol 49: 509-517, 2014.

14. Buergy D, Wenz F, Groden C and Brockmann MA: Tumor-platelet interaction in solid tumors. Int J Cancer 130: 2747-2760, 2012.

15. Gay LJ and Felding-Habermann B: Contribution of platelets to tumour metastasis. Nat Rev Cancer 11: 123-134, 2011.

16. Zhang X, Lv Z, Yu H and Zhu J: The clinicopathological and prognostic role of thrombocytosis in patients with cancer: A meta-analysis. Oncol Lett 13: 5002-5008, 2017

17. Guo T, Krzystanek M, Szallasi Z and Szallasi A: Thrombocytosis portends adverse prognostic significance in patients with stage II colorectal carcinoma. F1000Res 3: 180, 2014.

18. Chen M and Geng JG: P-selectin mediates adhesion of leukocytes, platelets, and cancer cells in inflammation, thrombosis, and cancer growth and metastasis. Arch Immunol Ther Exp (Warsz) 54: 75-84, 2006.

19. Ho-Tin-Noé B, Demers M and Wagner DD: How platelets safeguard vascular integrity. J Thromb Haemost 9 (Suppl 1): S56-S65, 2011

20. Nakashima J, Tachibana M, Horiguchi Y, Oya M, Ohigashi T, Asakura $\mathrm{H}$ and Murai M: Serum interleukin 6 as a prognostic factor in patients with prostate cancer. Clin Cancer Res 6: 2702-2706, 2000.

21. Paule B, Belot J, Rudant C, Coulombel C and Abbou CC: The importance of IL-6 protein expression in primary human renal cell carcinoma: An immunohistochemical study. J Clin Pathol 53: 388-390, 2000

22. Shen XM, Xia YY, Lian L, Zhou C, Li XL, Han SG, Zheng Y, Gong FR, Tao M, Mao ZQ and Li W: Mean platelet volume provides beneficial diagnostic and prognostic information for patients with resectable gastric cancer. Oncol Lett 12: 2501-2506, 2016.

23. Wodarczyk M, Kasprzyk J, Sobolewska-Wodarczyk A, Włodarczyk J, Tchórzewski M, Dziki A and Dziki Ł: Mean platelet volume as a possible biomarker of tumor progression in rectal cancer. Cancer Biomark 17: 411-417, 2016

24. Kurtoglu E, Kokcu A, Celik H, Sari S and Tosun M: Platelet indices may be useful in discrimination of benign and malign endometrial lesions, and early and advanced stage endometrial cancer. Asian Pac J Cancer Prev 16: 5397-5400, 2015.
25. Ma X, Wang Y, Sheng H, Tian W, Qi Z, Teng F and Xue F: Prognostic significance of thrombocytosis, platelet parameters and aggregation rates in epithelial ovarian cancer. J Obstet Gynaecol Res 40: 178-183, 2014

26. Karateke A, Kaplanoglu M and Baloglu A: Relations of platelet indices with endometrial hyperplasia and endometrial cancer. Asian Pac J Cancer Prev 16: 4905-4908, 2015.

27. Borsig L: The role of platelet activation in tumor metastasis. Expert Rev Anticancer Ther 8: 1247-1255, 2008.

28. Ay C, Pabinger I and Cohen AT: Cancer-associated venous thromboembolism: Burden, mechanisms, and management. Thromb Haemost 117: 219-230, 2017.

29. Kurt M, Onal IK, Sayilir AY, Beyazit Y, Oztas E, Kekilli M, Turhan N, Karaman K and Akdogan M: The role of mean platelet volume in the diagnosis of hepatocellular carcinoma in patients with chronic liver disease. Hepatogastroenterology 59: 1580-1582, 2012.

30. Karaman K, Bostanci EB, Aksoy E, Kurt M, Celep B, Ulas M, Dalgic T, Surmelioglu A, Hayran M and Akoglu M: The predictive value of mean platelet volume in differential diagnosis of non-functional pancreatic neuroendocrine tumors from pancreatic adenocarcinomas. Eur J Intern Med 22: e95-e98, 2011.

31. Cho SY, Yang JJ, You E, Kim BH, Shim J, Lee HJ, Lee WI, Suh JT and Park TS: Mean platelet volume/platelet count ratio in hepatocellular carcinoma. Platelets 24: 375-377, 2013.

32. Inagaki N, Kibata K, Tamaki T, Shimizu T and Nomura S: Prognostic impact of the mean platelet volume/platelet count ratio in terms of survival in advanced non-small cell lung cancer. Lung Cancer 83: 97-101, 2014.

33. Kumagai S, Tokuno J, Ueda Y, Marumo S, Shoji T, Nishimura T, Fukui $\mathrm{M}$ and Huang CL: Prognostic significance of preoperative mean platelet volume in resected non-small-cell lung cancer. Mol Clin Oncol 3: 197-201, 2015.

34. Tuncel T, Ozgun A, Emirzeoglu L, Celik S, Bilgi O and Karagoz B: Mean platelet volume as a prognostic marker in metastatic colorectal cancer patients treated with bevacizumab-combined chemotherapy. Asian Pac J Cancer Prev 15: 6421-6423, 2014

35. Li N, Yu Z, Zhang X, Liu T, Sun YX, Wang RT and Yu KJ: Elevated mean platelet volume predicts poor prognosis in colorectal cancer. Sci Rep 7: 10261, 2017.

36. Mutlu H, Berk V, Karaca H, Erden A, Aslan T and Akca Z: Treatment regimen with bevacizumab decreases mean platelet volume in patients with metastatic colon cancer. Clin Appl Thromb Hemost 18: 546-548, 2012.

37. Mutlu H, Artis TA, Erden A and Akca Z: Alteration in mean platelet volume and platicrit values in patients with cancer that developed thrombosis. Clin Appl Thromb Hemost 19: 331-333, 2013.

38. Vagdatli E, Gounari E, Lazaridou E, Katsibourlia E, Tsikopoulou F and Labrianou I: Platelet distribution width: A simple, practical and specific marker of activation of coagulation. Hippokratia 14: 28-32, 2010.

39. Hirahara N, Matsubara T, Kawahara D, Mizota Y, Ishibashi S and Tajima Y: Prognostic value of hematological parameters in patients undergoing esophagectomy for esophageal squamous cell carcinoma. Int J Clin Oncol 21: 909-919, 2016.

40. Song X, Zhu H, Pei Q, Tan F, Li C, Zhou Z, Zhou Y, Yu N, Li Y and Pei H: Significance of inflammation-based indices in the prognosis of patients with non-metastatic colorectal cancer. Oncotarget 8: 45178-45189, 2017.

This work is licensed under a Creative Commons Attribution-NonCommercial-NoDerivatives 4.0 International (CC BY-NC-ND 4.0) License. 